Энергетика. Изв. высш. учеб. заведений и энерг. объединений СНГ. Т. 62, № 6 (2019), с. 528-535

https://doi.org/10.21122/1029-7448-2019-62-6-528-535

УДК 621.311 .016

\title{
Математические модели
}

\section{и алгоритмы оптимального управления нагрузкой электропотребителей}

\author{
И. У. Рахмонов ${ }^{1)}$, К. М. Реймов ${ }^{2)}$ \\ ${ }^{1)}$ Ташкентский государственный технический университет имени Ислама Каримова \\ (Ташкент, Республика Узбекистан), \\ ${ }^{2)}$ Каракалпакский государственный университет (Нукус, Республика Узбекистан)
}

(C) Белорусский национальный технический университет, 2019

Belarusian National Technical University, 2019

Реферат. Один из основных путей обеспечения экономичных режимов работы энергосистем при краткосрочном планировании - выравнивание графиков нагрузок посредством оптимального управления электропотреблением. Выравнивание графиков нагрузок с целью снижения расходов можно осуществить привлечением потребителей на основе административных и экономических мер. Административные меры связаны с принудительным ограничением нагрузок потребителей в определенных интервалах периода планирования. Эти меры, с одной стороны, дают выигрыш для энергосистемы за счет выравнивания графика нагрузки, а с другой - приносят ущерб потребителям. В конечном итоге в целом для энергосистемы ущерб может оказаться больше, чем выигрыш. Поэтому их целесообразно использовать в условиях дефицита электроэнергии и мощности в энергосистеме. Оптимальное планирование краткосрочных режимов энергосистем по полученным после выравнивания жестким графикам нагрузок потребителей можно осуществить традиционными методами. Решение данной задачи первоначально следует провести в условиях нежестких графиков нагрузок, получаемых в результате директивного использования административно-экономических мер, осуществляемых за счет специально разработанных моделей. В статье предложены математическая модель задачи оптимизации графиков нагрузок регулируемых электропотребителей при оптимальном планировании краткосрочных режимов энергосистем, алгоритм оптимального планирования краткосрочного режима энергосистемы с оптимизацией графиков нагрузок регулируемых электропотребителей, а также алгоритмы учета простых и функциональных ограничений в виде равенств и неравенств при оптимизации графиков нагрузок электропотребителей. Эффективность алгоритма оптимизации краткосрочного режима энергосистемы с учетом оптимального управления нагрузкой регулируемых электропотребителей исследована на примере оптимального покрытия графика нагрузки энергосистемы, содержащей двух потребителей с регулируемыми графиками нагрузок, двумя ТЭС. На основе проведенных расчетно-экспериментальных исследований установлено, что предложенная математическая модель задачи является адекватной,

\begin{tabular}{ll}
\hline Адрес для переписки & Address for correspondence \\
Рахмонов Икромжон Усмонович & Rakhmonov Ikromzhon U. \\
Ташкентский государственный & Tashkent State \\
технический университет & Technical University \\
имени Ислама Каримова & named after Islam Karimov \\
ул. Университетская, 2a, & 2a Universitetskaya str., \\
100095, г. Ташкент, Республика Узбекистан & 100095, Tashkent, Republic of Uzbekistan \\
Тел.: +99890 945-87-00 & Tel.: +99890945-87-00 \\
ilider1987@yandex.ru & ilider1987@yandex.ru \\
\hline
\end{tabular}


разработанные алгоритмы оптимального планирования краткосрочных режимов энергосистем с оптимизацией графиков нагрузок регулируемых электропотребителей и учетом различных видов ограничений обладают высокими вычислительными качествами.

Ключевые слова: электроэнергетическая система, математическая модель, оптимизация режима, планирование, управление, цикл регулирования, выравнивание графика нагрузки, целевая функция, метод, алгоритм, ограничение, активная мощность, электропотребление, регулирование нагрузки

Для цитирований: Рахмонов, И. У. Математические модели и алгоритмы оптимального управления нагрузкой электропотребителей / И. У. Рахмонов, К. М. Реймов // Энергетика. Изв. высш. учеб. заведений и энерг. объединений СНГ. 2019. Т. 62, № 6. С. 528-535. https://doi.org/10.21122/1029-7448-2019-62-6-528-535

\title{
Mathematical Models \\ and Algorithms of Optimal Load Management of Electricity Consumers
}

\author{
I. U. Rakhmonov ${ }^{1)}$, K. M. Reymov ${ }^{2)}$ \\ ${ }^{1)}$ Tashkent State Technical University named after Islam Karimov (Tashkent, \\ Republic of Uzbekistan), \\ ${ }^{2}$ Karakalpak State University (Nukus, Republic of Uzbekistan)
}

\begin{abstract}
Load profile alignment based on optimal power consumption management is considered to be one of the main ways to ensure efficient operation of energy systems in the short-term planning. Alignment load profile with a view to reducing costs can be implemented with the aid of consumers' involvement by administrative and economic measures. Administrative measures are associated with the forced restriction of consumer loads in certain intervals of the planning period. On one hand, these measures provide benefits to the power system by alignment load profile, and on the other hand, they cause detriment to consumers. Ultimately, in some cases, for the whole power system, the detriment may be greater than the benefits. Therefore, it is advisable to use administrative measures in conditions of shortage of power and electricity in the power system. Optimal planning of short-term regimes of power systems according to rigid load profile received after alignment can be carried out by traditional methods. The solution of such a problem ought to be initially carried out under conditions of non-rigid load profile resulting from the directive use of administrative and economic measures carried out with the help of specially developed models. In this regard, the paper proposes a mathematical model of the problem of optimizing load profile of regulated electricity consumers to be used for optimal planning of shortterm power system modes, an algorithm for optimal planning of a short-term power system mode with optimizing load profile of regulated power consumers. Also, algorithms are proposed for accounting for simple and functional constraints in the form of equalities and inequalities when optimizing load profile. The effectiveness of the described algorithm for optimizing the short-term mode of the power system, taking into account the optimal load control of adjustable electricity consumers, has been studied using the example of optimal coverage of the load profile of power system, which contains two consumers with adjustable load profile, and two TPPs. Based on the calculated-and-experimental studies, it was determined that the proposed mathematical model of the problem is adequate, while the developed algorithms for optimal planning of short-term power system modes with optimization of load profile of regulated electricity consumers and taking into account various types of limitations are of high computational qualities.
\end{abstract}

Keywords: electric power system, mathematical model, mode optimization, planning, control, regulation cycle, load curve alignment, objective function, method, algorithm, limitation, active power, power consumption, load control

For citation: Rakhmonov I. U., Reymov K. M. (2019) Mathematical Models and Algorithms of Optimal Load Management of Electricity Consumers. Energetika. Proc. CIS Higher Educ. Inst. and Power Eng. Assoc. 62 (6), 528-535. https://doi.org/10.21122/1029-7448-2019-62-6-528-535 (in Russian) 


\section{Введение}

Развитие энергетики ряда стран СНГ, в частности Республики Узбекистан, на современном этапе сопровождается появлением резко неравномерных графиков нагрузок, частыми изменениями схем электрических сетей, постепенным переходом на рыночные формы функционирования и соответствующим усложнением управления режимами электроэнергетических систем (ЭЭС).

Резкая неравномерность графика нагрузки приводит к увеличению составляющих затрат на производство и соответствующему росту у потребителей издержек на покупку электроэнергии. Это, в частности, связано с эксплуатацией в электростанциях при максимальных нагрузках менее экономичного оборудования, использующего энергоресурсы недостаточно эффективно. Поэтому экономичные режимы энергосистемы при краткосрочном планировании могут быть обеспечены за счет выравнивания графика нагрузки посредством управления электропотреблением.

Выравнивание графика нагрузки энергосистемы можно осуществить привлечением потребителей на основе административных и экономических мер. Административные меры связаны с принудительным ограничением нагрузки потребителей в определенных интервалах периода планирования. Эти меры, с одной стороны, дают выигрыш для энергосистемы за счет выравнивания графика нагрузки, а с другой - приносят ущерб потребителям. В конечном итоге в некоторых случаях, ущерб может оказаться больше, чем выигрыш. Поэтому их целесообразно использовать в условиях дефицита электроэнергии и мощности в энергосистеме [1-3].

Выравнивание графика нагрузки энергосистемы на основе использования экономических мер в условиях функционирования рыночных механизмов управления режимами ЭЭС представляет большой интерес. Разработанные методики выравнивания графика нагрузки на базе экономических мер предусматривают в основном добровольную реакцию потребителей в ответ на изменение стоимости электроэнергии в течение планируемого периода пропорционально суммарной нагрузке, а также на компенсационные выплаты для стимулирования снижения потребления в часы максимальных и увеличения в часы минимальных нагрузок [4-6].

\section{Основная часть}

В условиях перехода на рыночные механизмы управления режимами энергосистем имеют место также административно-экономические меры выравнивания графиков нагрузок электропотребителей. При этом для некоторых крупных потребителей определяются не жесткие нагрузки для каждого интервала, а их минимально и максимально возможные значения при сохранении постоянного электропотребления за рассматриваемый период планирования. В таких условиях появляется возможность получения дополнительного экономического эффекта за счет оптимального планирования режимов энергосистемы с учетом нежестких графиков нагрузок потребителей. Часть получаемой дополнительной экономической прибыли при этом может быть израсходована для стимулирования потребителей за их участие в процессе оптимизации $[7,8]$. 
Оптимальное планирование краткосрочных режимов энергосистем по полученным после выравнивания жестким графикам нагрузок потребителей можно произвести традиционными методами. Решение этой задачи в условиях нежестких графиков нагрузок, получаемых в результате использования для выравнивания графиков нагрузок административно-экономических мер, может быть осуществлено на основе специально разработанных моделей $[9,10]$.

Задача оптимизации краткосрочных режимов энергосистем с оптимальным управлением нагрузкой электропотребителей математически описывается следующим образом:

- минимизировать целевую функцию, представляющую собой функцию суммарных топливных издержек на производство электроэнергии всеми ТЭС за цикл регулирования $T$ :

$$
B=\sum_{t \in T} \sum_{i \in T} B_{i}\left(P_{i}^{t}\right)
$$

- с учетом ограничений:

- по балансу мощности в энергосистеме в каждом интервале цикла регулирования $T$

$$
W_{t}=\sum_{i \in N} P_{i}^{t}-\sum_{i \in M} P_{j}^{t}=0, t \in T
$$

- по балансу электроэнергии за цикл регулирования для каждого из расчетных (участвующих в оптимизации) электропотребителей

$$
\varphi_{j}=\sum_{t \in T} P_{j}^{t}-\Im_{j}^{T}=0, j \in M ;
$$

- по минимально и максимально возможным нагрузкам расчетных электростанций и электропотребителей:

$$
\begin{aligned}
& P_{i}^{t, \text { min }} \leq P_{i}^{t} \leq P_{i}^{t, \text { max }}, i \in N ; t \in T ; \\
& P_{j}^{t, \text { min }} \leq P_{j}^{t} \leq P_{j}^{t, \text { max }}, i \in M ; t \in T ;
\end{aligned}
$$

- по перетокам мощностей контролируемых линий электропередач (ЛЭП)

$$
P_{l}^{t} \leq \bar{P}_{l}^{t}, l \in L_{p} ; t \in T,
$$

где $N, M$ - множество ТЭС и электропотребителей, участвующих в оптимизации; $L_{p}-$ множество ЛЭП, в которых контролируются перетоки мощности; $P_{i}^{t}, P_{j}^{t}$ - мощность $i$-й ТЭС и $j$-го электропотребителя в $t$-м интервале цикла регулирования; $B_{i}\left(P_{i}^{t}\right)$ - топливная издержка $i$-й ТЭС при ее нагрузке в $t$-м интервале цикла регулирования; $P_{i}^{t}, W_{t}, \varphi_{j}$ - функции небалансов активной мощности в $t$-м интервале и электроэнергии $j$-го потребителя за цикл регулирования $T$ соответственно.

Предлагаемый алгоритм решения рассматриваемой задачи предусматривает учет ограничений в виде равенства разделением переменных мощ- 
ностей расчетных станций и нагрузок регулируемых электропотребителей на независимые $\left(y_{i}, i=1,2, \ldots, T+m\right)$ и зависимые $\left(x_{j}, j=1,2, \ldots, T^{*}(n+m)-\right.$ $-(T+m))$ переменные. При этом из каждого ограничения в виде равенства по одной из переменных выделяется в качестве зависимой переменной. В результате число зависимых переменных равняется числу ограничений в виде равенства. За такие переменные рекомендуется выбрать переменные с большим допустимым диапазоном изменения.

Целевая функция представляется как

$$
F=B+\sum_{i \in T} \sum_{l \in L_{p}} \amalg_{l}^{t},
$$

где $\amalg_{l}^{t}$ - штрафная функция, учитывающая ограничения по перетоку мощности $l$-й контролируемой ЛЭП в $t$-м интервале.

Расчетный процесс начинается с принятия начальных значений для независимых переменных, начальных шагов и точности расчета. Величины зависимых переменных при известных значениях независимых переменных находятся на основе решения системы уравнений, состоящей из ограничений в виде равенств (2) и (3).

Компоненты градиента целевой функции (7) вычисляются с учетом сложной зависимости целевой функции от независимых переменных

$$
\frac{\partial F^{(k-1)}}{\partial y_{j}}=\frac{\partial \stackrel{-}{F}^{(k-1)}}{\partial y_{j}}+\sum_{i=1}^{T+m} \frac{\partial \stackrel{-}{F}^{(k-1)}}{\partial x_{i}} \frac{\partial x^{(k-1)}}{\partial y_{j}}, j=1,2, \ldots, T^{*}(n+m)-(T+m) .
$$

Независимые переменные на каждой $k$-й итерации вычисляются по формуле

$$
y_{j}^{(k)}=y_{j}^{(k-1)}-h_{j}^{(k)} \frac{\partial F^{(k-1)}}{\partial y_{j}}, j=1,2, \ldots, T^{*}(n+m)-(T+m)
$$

Шаги в направлении антиградиента определяются по условиям:

$$
h^{t(h)}=\left\{\begin{array}{lll}
1,3 h^{t(k-1)}, & \text { если } & \frac{\partial F^{(k-1)}}{\partial P^{t}} \frac{\partial F^{(k-2)}}{\partial P^{t}}>0 ; \\
0,4 h^{t(k-1)}, & \text { если } & \frac{\partial F^{(k-1)}}{\partial P^{t}} \frac{\partial F^{(k-2)}}{\partial P^{t}}<0 .
\end{array}\right.
$$

Таким образом, процесс расчета по данному алгоритму оптимизации краткосрочного режима энергосистемы с оптимальным управлением нагрузкой электропотребителей и учетом ограничений выделением зависимых переменных выглядит следующим образом:

1) принимаются начальные значения мощностей расчетных станций и нагрузочных узлов с регулируемыми электропотребителями, которые являются независимыми переменными, шаги по независимым переменным и точность расчета; 
2) на основе решения системы уравнений, состоящей из ограничений в виде (2) и (3), при начальных значениях независимых переменных определяются значения мощностей расчетных станций и нагрузочных узлов, принятых как зависимые переменные;

3) по (8) вычисляются компоненты градиента целевой функции по независимым переменным;

4) из условия (10) определяются шаги в направлении антиградиента по независимым переменным;

5) по (9) определяются значения независимых переменных в очередной итерации;

6) на основе решения системы уравнений, состоящей из ограничений в виде (2) и (3), при полученных значениях независимых переменных находят значения зависимых переменных в очередной итерации;

7) проверяется обеспечение условия сходимости итеративного процесса $\left|B^{(k-1)}-B^{(k)}\right| \leq \varepsilon_{B}$ при выполнении всех ограничений.

При выполнении условия сходимости расчетный процесс останавливается и результат, полученный на последней итерации, принимается как оптимальный. В противном случае аналогично выполняется следующая итерация, начиная с пункта 3.

Эффективность описанного алгоритма исследована, в частности, на примере оптимального покрытия графика нагрузки энергосистемы, содержащей двух потребителей с регулируемыми графиками нагрузок, двумя ТЭС. Характеристики топливных издержек станций представлены в квадратичной форме как $B_{i}=a_{i}+b_{i} P_{i}+c_{i} P_{i}^{2}$ (дол./ч), коэффициенты которой приведены в табл. 1.

Таблица 1

Коэффициенты энергетических характеристик ТЭС ( $\left.a_{i}=0\right)$

The coefficients of power-generating characteristics of thermal power plants $\left(a_{i}=0\right)$

\begin{tabular}{|c|c|c|}
\hline ТЭС & $b_{i}$ & $c_{i}$ \\
\hline TЭС-1 & 4,0 & 0,024 \\
\hline TЭС-2 & 6,0 & 0,016 \\
\hline
\end{tabular}

Электроэнергия, получаемая потребителями в течение цикла регулирования, и их нежесткие графики нагрузок даны в табл. 2.

Таблииа 2

Графики граничных нагрузок и электроэнергия, отпускаемая потребителям в течение полного цикла регулирования

Border load profiles and electricity supplied to consumers during the full regulatory cycle

\begin{tabular}{|c|c|c|c|c|c|}
\hline \multirow{2}{*}{ Потребитель } & \multirow{2}{*}{$\begin{array}{l}\text { Получаемая } \\
\text { электроэнергия, } \\
\text { МВт·ч }\end{array}$} & \multicolumn{4}{|c|}{ Граничная (минимально и максимально возможная) } \\
\cline { 3 - 6 } & \multicolumn{4}{|c|}{ нагрузка по интервалам, МВт } \\
\hline \multirow{2}{*}{1} & \multirow{2}{*}{1650} & 300 & 400 & 3 & 4 \\
\hline \multirow{2}{*}{2} & \multirow{2}{*}{1400} & 400 & 400 & 380 & 470 \\
\cline { 3 - 6 } & & 200 & 350 & 450 & 250 \\
\cline { 3 - 6 } & & 300 & 350 & 600 & 300 \\
\hline
\end{tabular}


Результаты оптимального планирования режима энергосистемы с учетом управления графиков нагрузок потребителей за рассматриваемый период приведены в табл. 3 .

Оптимальное покрытие нежестких графиков нагрузок

Таблица 3 Optimal coverage of non-rigid load profile

\begin{tabular}{|c|c|c|c|c|c|c|c|}
\hline \multirow{2}{*}{\multicolumn{2}{|c|}{ Субъект }} & \multirow{3}{*}{$\begin{array}{c}\text { Отпускаемая } \\
\text { электроэнергия, } \\
\text { МВт.ч }\end{array}$} & \multicolumn{4}{|c|}{$\begin{array}{c}\text { Оптимальный график нагрузок } \\
\text { потребителей и ТЭС, МВт }\end{array}$} & \multirow{3}{*}{ 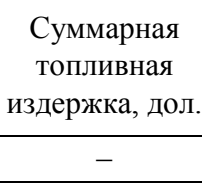 } \\
\hline & & & \multirow{2}{*}{$\begin{array}{c}1 \\
400\end{array}$} & \multirow{2}{*}{$\begin{array}{c}2 \\
400\end{array}$} & \multirow{2}{*}{$\frac{3}{350}$} & \multirow{2}{*}{$\begin{array}{c}4 \\
500 \\
\end{array}$} & \\
\hline Потре- & 1 & & & & & & \\
\hline битель & 2 & 1400 & 300 & 350 & 450 & 300 & - \\
\hline \multirow{2}{*}{ ТЭС } & ТЭС-1 & 1320 & 305 & 325 & 345 & 345 & 15760,8 \\
\hline & ТЭС-2 & 1730 & 395 & 425 & 455 & 455 & 22391,2 \\
\hline \multicolumn{2}{|c|}{$\begin{array}{l}\text { Суммарный } \\
\text { по энергосистеме }\end{array}$} & 3050 & 700 & 750 & 800 & 800 & 38152,0 \\
\hline
\end{tabular}

Для сравнения результатов оптимального планирования режима энергосистемы приведены результаты оптимального покрытия жестких (нерегулируемых) графиков граничных нагрузок потребителей. При этом суммарные топливные издержки составляли 39160,0 и 38169,3 дол., которые соответственно большие, чем при планировании с оптимальным управлением нагрузок электропотребителей.

\section{ВЫВОДЬ}

1. Разработаны математическая модель и алгоритм оптимального планирования краткосрочных режимов энергосистем с управлением нагрузкой электропотребителей. В результате создана возможность определения оптимальных нагрузок расчетных электростанций и регулируемых электропотребителей с учетом режимных и технологических ограничений и обеспечения на их основе эффективных режимов работы энергосистемы в допустимой области.

2. В условиях нежестких графиков нагрузок потребителей можно получить дополнительный экономический эффект при оптимальном планировании режима энергосистемы. При этом жесткие оптимальные графики нагрузок потребителей, которые должны быть сохранены, определяются в результате оптимизации.

\section{ЛИТЕРАТУРА}

1. Руденко, Ю. Н. Автоматизация диспетчерского управления в электроэнергетике / Ю. Н. Руденко, В. А. Семенова. М.: МЭИ, 2000. 648 с.

2. Rahmonov, I. U. Analysis of Problems of Management of a Power Consumption and Ways of their Decisions / I. U. Rahmonov // Science and Innovation in the XXI century: Problems and Solutions. Collection of Conference Papers of International Scientific-Practical Conference (29.09.2015, the United Kingdom, London). London, 2015. P. 22-25. 
3. Лазуренко, А. П. Определение потенциального экономического эффекта от выравнивания графика электрической нагрузки ОЭС Украины / А. П. Лазуренко, Г. И. Черкашина // Світлотехніка та електроенергетика. 2009. № 1. С. 4-12.

4. Gayibov, T. Optimization of Modes of Electrical Power Systems with the Control of Power Consumption / T. Gayibov, K. Reymov // Proceedings of the $7^{\text {th }}$ International Scientific Symposium on Electrical Power Engineering, Elektroenergetika 2013, Sept., 2013. Stara Lesna, Slovakia, 2013. P. 83-85.

5. Аюев, Б. И. Управление электропотреблением: административные и экономические методы / Б. И. Аюев // Энергорынок. 2007. № 4. С. 12-17.

6. Gayibov, T. Sh. Optimal Planning of Short-Term Modes of Power Systems with Control of Loads of Electric Consumers and Taking into Account of Network Factor / T. Sh. Gayibov, K. M. Reymov // European Science Review. 2017. Vol. 3, No 9-10. P. 86-91.

7. Using Real-Time Control of Demand to Help Balance Generation and Load / B. A. Brooks [et al.] // IEEE Power Energy Magazine. 2010. Vol. 8, No 3. P. 20-29.

8. Management and Control of Domestic Smart Grid Technology / A. Molderink [et al.] // IEEE Trans. Smart Grid. 2010. Vol. 1, No 2. P. 109-119. https://doi.org/10.1109/tsg.2010. 2055904.

9. An Approach of DSM Techniques for Domestic Load Management Using Fuzzy Logic / P. Ravibabu [et al.] // 2009 IEEE International Conference on Fuzzy Systems. Proceedings of a Meeting held 20-24 August 2009, Jeju Island, Korea. Institute of Electrical and Electronics Engineers. 2009. P. 1303-1307. https://doi.org/10.1109/fuzzy.2009.5277401.

10. Соколов, В. К. Задачи оперативной оптимизации производства электроэнергии в условиях рыночных отношений / В. К. Соколов, А. А. Прейгель, В. Г. Васильев // Электричество. 2007. № 1. С. 2-9.

Поступила 19.02.2019 Подписана в печать 24.04.2019 Опубликована онлайн 29.11.2019

\section{REFERENCES}

1. Rudenko Yu. N., Semenova V. A. (2000) Automation of Dispatching Control in Electric Power Industry. Moscow, MEI Publ. 648 (in Russian).

2. Rahmonov I. U. (2015) Analysis of Problems of Management of a Power Consumption and Ways of their Decisions. Science and Innovation in the XXI century: Problems and Solutions. Collection of Conference Papers of International Scientific-Practical Conference (29.09.2015, the United Kingdom, London). London, 22-25.

3. Lazurenko A. P., Cherkashina G. I. (2009) Determination of the Potential Economic Effect of Leveling the Electrical Load Graph of the UES of Ukraine. Svitlotehnika ta Elektroenergeti$k a=$ Lighting Engineering \& Power Engineering, (1), 4-12 (in Russian).

4. Gayibov T., Reymov K. (2013) Optimization of Modes of Electrical Power Systems with the Control of Power Consumption. Proceedings of the $7^{\text {th }}$ International Scientific Symposium on Electrical Power Engineering, Elektroenergetika 2013, Sept., 2013. Stara Lesna, Slovakia, 83-85.

5. Ayuev B. I. (2007) Management of Electrical Consumption: Administrative and Economic Methods. EnergoRynok [Energy Market], (4), 12-17 (in Russian).

6. Gayibov T. Sh., Reymov K. M. (2017) Optimal Planning of Short-Term Modes of Power Systems with Control of Loads of Electric Consumers and Taking into Account of Network Factor. European Science Review. 3 (9-10), 86-91.

7. Brooks B. A., Lu E., Reicher D., Spirakis C., Weihl B. (2010) Using Real-Time Control of Demand to Help Balance Generation and Load. IEEE Power Energy Magazine. 8 (3), 20-29.

8. Molderink A., Bakker V., Bosman M., Hurink J., Smit G. (2010) Management and Control of Domestic Smart Grid Technology. IEEE Transactions on Smart Grid, 1 (2), 109-119. https://doi.org/10.1109/tsg.2010.2055904.

9. Ravibabu P., Praveen A., Chandra C., Reddy P., Teja M. (2009) An Approach of DSM Techniques for Domestic Load Management Using Fuzzy Logic. 2009 IEEE International Conference on Fuzzy Systems. Proceedings of a Meeting Held 20-24 Aug. 2009, Jeju Island, Korea. Institute of Electrical and Electronics Engineers, 1303-1307. https://doi.org/10.1109/fuzzy. 2009.5277401.

10. Sokolov V. K., Preigel A. A., Vasil'ev V. G. (2007) Objectives of Operational Optimization of Electricity Production in the Conditions of Market Relations. Elektrichestvo, (1), 2-9 (in Russian).

Received: 19 February 2019 Accepted: 24 April 2019 Published online: 29 November 2019 